\title{
CALL Score and RAS Score as Predictive Models for Coronavirus Disease 2019
}

Sultan M. Kamran ${ }^{1}$, Zill-e-Humayun Mirza ${ }^{1}$, Hussain Abdul Moeed ${ }^{2}$, Arshad Naseem ${ }^{3}$, Maryam Hussain 1 , Imran Fazal Sr. ${ }^{4}$, Farrukh Saeed ${ }^{5}$, Wasim Alamgir ${ }^{6}$, Salman Saleem ${ }^{7}$, Sidra Riaz ${ }^{4}$

1. Pulmonology, Pak Emirates Military Hospital, Rawalpindi, PAK 2. Internal Medicine, Pak Emirates Military Hospital, Rawalpindi, PAK 3. Critical Care, Pak Emirates Military Hospital, Rawalpindi, PAK 4. Medicine, Pak Emirates Military Hospital, Rawalpindi, PAK 5. Gastroenterology, Pak Emirates Military Hospital, Rawalpindi, PAK 6. Neurology, Pak Emirates Military Hospital, Rawalpindi, PAK 7. Infectious Disease, Pak Emirates Military Hospital, Rawalpindi, PAK

Corresponding author: Sultan M. Kamran, sultanmajoka79@hotmail.com

\begin{abstract}
Background: Coronavirus disease 2019 (COVID-19) is a novel infectious disease of multi-system involvement with significant pulmonary manifestations. So far, many prognostic models have been introduced to guide treatment and resource management. However, data on the impact of measurable respiratory parameters associated with the disease are scarce.
\end{abstract}

Objective: To demonstrate the role of Comorbidity-Age-Lymphocyte count-Lactate dehydrogenase (CALL) score and to introduce Respiratory Assessment Scoring (RAS) model in predicting disease progression and mortality in COVID-19.

Methodology: Data of 252 confirmed COVID-19 patients were collected at Pak Emirates Military Hospital (PEMH) from 10th April 2020 to 31st August 2020. The CALL score and proposed factors of RAS model, namely respiratory rate, oxygen saturation at rest, alveolar arterial gradient and minimal exercise desaturation test, were calculated on the day of admission. Progression of disease was defined and correlated with measured variables. Univariate and multivariate Cox regression analysis for each variable, its hazard ratio (HR) and 95\% confidence interval (CI) were calculated, and a nomogram was made using the high-risk respiratory parameters to establish the RAS model.

Results: Progression of disease and death was observed in 124 (49.2\%) and 49 (19.4\%) patients, respectively. Presence of more than $50 \%$ of chest infiltrates was significantly associated with worsening disease and death (p-value $<0.001$ ). Death was observed in $100 \%$ of patients who had critical disease category on presentation. Regression analysis showed that the presence of comorbidity (n: 180), in contrast to other variables of CALL score, was not a good prognosticator of disease severity (p-value: 0.565). Nonetheless, the CALL model itself was validated to be a reliable prognostic indicator of disease progression and mortality. Some 10 feet oxygen desaturation test (HR: 0.99, 95\%CI: 0.95-1.04, p-value: 0.706) was not a powerful predictor of the progression of disease. However, respiratory rate of more than 30 breaths/minute (b/m) (HR: 3.03, 95\%CI: 1.77-5.19), resting oxygen saturation of less than $90 \%$ (HR: $2.41,95 \% \mathrm{CI}: 1.15-5.06)$, and an elevated alveolar-arterial oxygen gradient (HR: 2.14 , 95\%CI: 1.04-4.39) were considered statistically significant highrisk predictors of disease progression and death, in the formed RAS model. The model resulted in $85 \%$ (95\%CI: 80\%-89\%) of area under the receiver operating characteristic curve (AUROC), with substantial positive (76\%, 95\% CI: 68\%-83\%) and negative predictive values (80\%, 95\% CI: $73 \%-87 \%$ ) for a cutoff value of seven. Patients with higher CALL and RAS scores also resulted in higher mortality.

Conclusion: CALL and RAS scores were strongly associated with progression and mortality in patients with COVID-19.

Categories: Internal Medicine, Infectious Disease, Pulmonology

Keywords: covid-19, prediction model, call score, prognostic markers, respiratory rate, oxygen saturation, a-a gradient, minimal exercise desaturation, comorbidities

\section{Introduction}

The novel coronavirus, severe acute respiratory syndrome coronavirus 2 (SARS-CoV-2), first described in December 2019 has spread to over 210 countries. The World Health Organization (WHO) announced the coronavirus disease 2019 (COVID-19) a pandemic on 11th March 2020 and up till now over 40 million substantiated cases of this illness have been recorded, with more than one million deaths [1]. COVID-19 is a virulent multi-system disease manifesting primarily as a respiratory illness with symptoms ranging from fever, cough, fatigue, sore throat and shortness of breath, to impending critical disease requiring mechanical ventilation, or ending in death [2]. People with underlying conditions such as hypertension (HTN), cardiovascular disease, diabetes mellitus (DM), chronic obstructive pulmonary disease (COPD), and malignancy have an increased probability of advancement to severity and greater mortality [3]. Considering 
high fatality (61.5\%) in critically ill patients with SARS-CoV-2 infection [4], it is a matter of utmost concern to identify mild and moderate cases earlier and predict disease progression based on risk factors so that an early escalation of treatment is ensured.

Various predictive models for COVID-19 have been designed during this pandemic, which are now accessible in academic literature to aid medical decision making [5]. A predictive model, Comorbidity-Age-Lymphocyte count-Lactate dehydrogenase (CALL), has been devised to anticipate disease progression [6]. However, the CALL score does not cater for respiratory assessment parameters, which are also paramount in predicting disease progression and mortality [7]. It has been observed that around $15 \%$ of cases of COVID-19 are complicated by interstitial pneumonia and a variable degree of respiratory failure [2].

Early disclosure of increasing respiratory involvement of COVID-19 can help physicians to enact necessary measures including treatment escalation. For this purpose, an alternative model called the Respiratory Assessment Scoring (RAS) was devised in this study. Various easy-to-calculate clinical and laboratory respiratory parameters, such as respiratory rate (RR), resting oxygen $(\mathrm{O} 2)$ saturation, alveolar-arterial oxygen gradient (A-a gradient), and minimal exercise desaturation test were employed in order to generate potential high-risk factors to formulate the scoring model.

\section{Materials And Methods Study design and setting}

This single-center cross-sectional study was carried out at the department of pulmonology of the Pak Emirates Military Hospital (PEMH), Rawalpindi, Pakistan from 1st April 2020 to 31st August 2020 after approval by the institutional ethical review committee (ERC). Data of 252 hospitalized patients afflicted with COVID-19 admitted in the pulmonology ward were included. Informed consent was given by all subjects.

\section{Inclusion and exclusion criteria}

Inclusion criteria included: (1) COVID-19 diagnosed by real time-polymerase chain reaction (RT-PCR) positivity for SARS-CoV-2, (2) mild disease with comorbidities, (3) moderate, severe, and critical illness, (4) 10-80 years of age, and (5) both genders. Exclusion criteria comprised: (1) asymptomatic or mild disease with no comorbidity, (2) cases who expired within 24 hours of hospitalization, (3) advanced malignancies with life expectancy less than six months, and (4) presence of co-infections.

\section{Study definitions}

On admission, patients were stratified into disease categories based on criteria set by WHO [8]. Mild disease meant patients with uncomplicated upper respiratory tract viral infection having nonspecific manifestations such as low-grade fever, cough, body aches, muscle pain, sore throat, nasal congestion, headache, diarrhea, nausea, vomiting, anosmia, and ageusia. Moderate disease was defined as a case with lung infiltrates less than $50 \%$ of the total lung fields on X-ray chest and peripheral ground-glass opacities (GGOs) on highresolution computed tomography (HRCT) chest, and no evidence of hypoxemia or severe pneumonia. Severe disease was operationally described as COVID-19 pneumonia with evidence of hypoxemia (RR more than 30 breaths/minute $(\mathrm{b} / \mathrm{m})$ or partial pressure of oxygen $(\mathrm{PaO} 2)$ less than $80 \mathrm{mmHg}$ on arterial blood gases (ABGs) or lung infiltrates being over $50 \%$ of the lung fields). Critical disease was determined if there was evidence of either respiratory failure ( $\mathrm{PaO} 2<60 \mathrm{mmHg}$ ) or multiorgan dysfunction syndrome (MODS) measured by the sequential organ failure assessment (SOFA) score of over 10 or septic shock (systolic blood pressure less than $90 \mathrm{mmHg}$ or less than $40 \mathrm{mmHg}$ of baseline in hypertensive or urine output less than $0.5 \mathrm{~mL} / \mathrm{kg} / \mathrm{hour}$ ). Comorbidity was described as any chronic health issue for which the patient was using medications previously. Progression of the disease meant an increasing demand for oxygen and/or ascending up to higher disease severity category or death because of COVID-19 related complications.

\section{Data structure and collection}

As per institutional approach, every subject with comorbidities or moderate, severe and critical illness at the time of admission and during hospitalization underwent monitoring and evaluation. This process included, but was not restricted to, vital signs monitoring (including pulse oximetry), baseline laboratory testing (complete blood count, coagulation profile, liver and renal function tests), blood testing for cytokine release storm [ferritin, D-dimers, lactate dehydrogenase (LDH), interleukin-6 (IL-6), absolute lymphocyte count (ALC)], tests for cardiac injury biomarkers [troponins, creatine kinase-MB (CKMB)], HRCT chest, and ABGs analysis. Respiratory specimens were also investigated for co-infections (influenza, avian influenza, respiratory syncytial virus, adenovirus, parainfluenza) via PCR analysis. During the stay in hospital, many of the above parameters were repeated when deemed necessary. A 10 feet walk $\mathrm{O} 2$ desaturation test was devised for minimal exercise desaturation assessment considering patient safety and disease condition since many already known exercise tests were deemed excessive for admitted patients [9]. The 10 feet walk $\mathrm{O} 2$ desaturation test was carried out under supervised care and only after the patient's consent. An oxygen desaturation of equal to and greater than three percent was notable for this test.

Extracted data of all the subjects included in the study comprised of variables such as age, gender, 


\section{Cureus}

comorbidities, contact history, types of symptoms, HRCT chest pattern, percentage of lung involvement, and initial disease category. For estimation of the CALL score added information, including ALC and LDH levels at the day of admission (day zero) were obtained. For respiratory assessment, variables such as RR, $\mathrm{O} 2$ saturation with pulse oximetry at rest and supported by ABGs, A-a gradient and 10 feet walk O2 desaturation test results were retrieved for day zero. To note the progression of illness, variables such as the final disease category and mortality were noted.

\section{Statistical analysis}

Data were analyzed using R software version 4.0.2 (R Foundation for Statistical Computing, Vienna, Austria). Continuous variables were checked for skewness with the Shapiro-Wilk test [10] and were expressed as medians [interquartile range (IQR)] and compared using the Mann-Whitney test. Categorical variables were presented as numbers (percentage) and correlated by the chi-square test. All statistical results were deemed significant at a $p$-value $<0.05$. Univariate and multivariate Cox regression analysis was used for assessing the relevance of each variable of the CALL score and proposed respiratory parameters of the RAS model to verify and investigate, respectively, the high-risk factors for progression of illness with their hazard ratio (HR) and 95\% confidence interval (95\% CI). Respiratory parameters that were statistically significant after multivariate Cox analysis, were elected for formation of a nomogram which proportionally converted each multivariate regression coefficient to a 0 -100 point scale.

\section{Model formation}

The RAS model was established with scoring points allotted from 1 to 10 after the above multivariate regression was utilized to determine the relative points for each significant respiratory parameter to reflect their impact on the progression of disease. The prognostic parameters were classified based on normal range and values for $\mathrm{RR}$ and resting $\mathrm{O} 2$ saturation while for A-a gradient an elevated value with respect to normal for age was considered noteworthy [11], as outlined in Table 1.

\begin{tabular}{|c|c|}
\hline & Points \\
\hline \multicolumn{2}{|c|}{ Respiratory rate (b/m) } \\
\hline$\leq 20$ & 1 \\
\hline $21-25$ & 2 \\
\hline $26-30$ & 3 \\
\hline$>30$ & 4 \\
\hline \multicolumn{2}{|c|}{ Resting oxygen saturation (\%) } \\
\hline$\geq 95$ & 1 \\
\hline $90-94$ & 2 \\
\hline$<90$ & 3 \\
\hline \multicolumn{2}{|c|}{ A-a Gradient } \\
\hline Normal & 1 \\
\hline Elevated & 3 \\
\hline
\end{tabular}

\section{TABLE 1: RAS calculator.}

RAS, respiratory assessment scoring; b/m, breaths/minute; A-a Gradient, alveolar-arterial oxygen gradient.

In addition, receiver operating characteristic (ROC) curves were used to evaluate the performance of the RAS prognostic model along with computation of area under the ROC (AUROC). Optimal cutoff values for progression and mortality of disease were also calculated and assessed by sensitivity, specificity, predictive values, and likelihood ratios.

\section{Results}

\section{Clinical characteristics}

Overall, 252 COVID-19 patients were entered in the study, median age 59 (IQR: 50-70), among which the majority were males (86.5\%). Out of these 252 cases, 180 (71.4\%) had comorbidities which mainly included 
hypertension (32.8\%), diabetes mellitus (30.6\%), and cardiovascular disease (23.3\%). History of contact with confirmed COVID-19 cases was noted in 69 (27.4\%) patients. Among the reported symptoms cough (78.2\%) was the commonest, followed by fever (75.8\%), shortness of breath (66.3\%), body aches (20.2\%), and diarrhea (7.9\%). Progression of illness was noted in $60 \%$ of patients who originally presented with shortness of breath ( $\mathrm{p}<0.001)$. HRCT chest showed classic findings for COVID-19 in 181 (71.8\%) cases, while 23 (9.1\%) had atypical and 24 (9.5\%) had mixed findings. In 134 (53.2\%) patients, lung involvement was over 50\%, of which $70.9 \%$ worsened and $28.4 \%$ expired ( $\mathrm{p}<0.001$ ). Based on established categories, at admission 115 (45.6\%) patients were classified to have severe disease, 99 (39.3\%) moderate, 22 (8.7\%) critical disease, and only 16 (6.3\%) to mild group with comorbidities. Overall, progression of the disease appeared in 124 (49.2\%) cases who consisted mainly of patients with severe disease category (63\%), followed by patients with critical disease (17.7\%), moderate disease (14.5\%), and a few with mild disease with comorbidities (4.8\%). Some $100 \%$ of patients in our study with critical initial disease category eventually died. Clinical attributes of patients were studied among stable, worsened and mortality associated groups, values of which are revealed in Table 2.

\begin{tabular}{|c|c|c|c|c|c|c|}
\hline & Overall (n = 252) & Stable $(n=128)$ & Worsened $(n=124)$ & p-Value & Death $(n=49)$ & p-Value \\
\hline Age, years & $59(50-70)$ & $56(48-68)$ & $62(53-72)$ & 0.005 & $70(58-75)$ & 0.005 \\
\hline Comorbidity & $180(71.4)$ & $92(71.9)$ & $88(71)$ & 0.873 & $43(87.8)$ & 0.005 \\
\hline Diabetes & $55(21.8)$ & $28(21.9)$ & $27(21.8)$ & 0.985 & $9(18.4)$ & 0.514 \\
\hline Hypertension & $59(23.4)$ & $33(25.8)$ & $26(21)$ & 0.367 & $11(22.4)$ & 0.859 \\
\hline Cardiovascular disease & $42(16.7)$ & 23 (18) & 19 (15.3) & 0.573 & 7 (14.3) & 0.618 \\
\hline Asthma/COPD & $9(3.6)$ & $5(3.9)$ & $4(3.2)$ & 0.771 & $4(8.2)$ & 0.054 \\
\hline CLD & $3(1.2)$ & $0(0)$ & $3(2.4)$ & 0.077 & $2(4)$ & 0.038 \\
\hline CRF & $2(0.8)$ & $1(0.8)$ & $1(0.8)$ & 0.982 & $1(2)$ & 0.273 \\
\hline Fever & $191(75.8)$ & $87(68)$ & 104 (83.9) & 0.003 & $42(85.7)$ & 0.071 \\
\hline Cough & $197(78.2)$ & $96(75)$ & 101 (81.5) & 0.215 & $38(77.6)$ & 0.906 \\
\hline Dyspnea & $167(66.3)$ & 66 (51.6) & $101(81.5)$ & $<0.001$ & $43(87.8)$ & $<0.001$ \\
\hline Lymphocyte count, $\times 10^{9} / \mathrm{L}$ & $1.2(0.7-1.5)$ & $1.3(0.9-1.6)$ & $0.8(0.7-1.3)$ & $<0.001$ & $0.8(0.7-1.1)$ & $<0.001$ \\
\hline LDH, U/L & 440 (289-789) & $358(231-554)$ & $592(365-928)$ & $<0.001$ & 678 (440-1009) & $<0.001$ \\
\hline Respiratory rate, b/m & $20(16-31)$ & $17(13-21)$ & $30(21-33)$ & $<0.001$ & $33(31-36)$ & $<0.001$ \\
\hline Restıng $\mathrm{O}_{2}$ saturation, \% & $92(86-96)$ & $94(92-97)$ & $88(82-92)$ & $<0.001$ & $81(79-85)$ & $<0.001$ \\
\hline $\mathrm{A}$-a gradient, $\mathrm{mmHg}$ & $39(12-76)$ & $17(9-39)$ & $69(39-98)$ & $<0.001$ & 80 (48-107) & $<0.001$ \\
\hline 10 teet $\mathrm{O}_{2}$ desaturation test, $\%$ & $6(2-11)$ & $3(1-7)$ & $9(4-14)$ & $<0.001$ & $15(9-16)$ & $<0.001$ \\
\hline Initial disease category & & & & $<0.001$ & & $<0.001$ \\
\hline Mild with comorbidities & $16(6.3)$ & $10(7.8)$ & $6(4.8)$ & & $2(4)$ & \\
\hline Moderate & 99 (39.3) & $81(63.6)$ & $18(14.5)$ & & $4(8.2)$ & \\
\hline Severe & $115(45.6)$ & $37(29)$ & $78(62.9)$ & & $21(42.9)$ & \\
\hline Critical & $22(8.7)$ & $0(0)$ & $22(17.7)$ & & $22(44.9)$ & \\
\hline HRCT chest findings & & & & 0.074 & & 0.331 \\
\hline Normal & $24(9.5)$ & $18(14)$ & $6(4.8)$ & & $2(4)$ & \\
\hline Typical & $181(71.8)$ & $86(67.2)$ & 95 (76.6) & & 35 (71.4) & \\
\hline Atypical & $23(9.1)$ & $13(10.2)$ & $10(8)$ & & $5(10.2)$ & \\
\hline Mixed & $24(9.5)$ & $11(8.6)$ & $13(10.5)$ & & 7 (14.3) & \\
\hline Lung involvement & & & & $<0.001$ & & $<0.001$ \\
\hline Normal & 21 (8.3) & $17(13.3)$ & $4(3.2)$ & & $1(2)$ & \\
\hline
\end{tabular}




\section{Cureus}

\begin{tabular}{|c|c|c|c|c|c|c|}
\hline$<50 \%$ & $97(38.5)$ & $72(56.3)$ & 25 (20.2) & & $10(20.4)$ & \\
\hline$>50 \%$ & $134(53.2)$ & 39 (30.5) & 95 (76.6) & & 38 (77.6) & \\
\hline Age & & & & $<0.001$ & & $<0.001$ \\
\hline$\leq 60$ years & $130(51.6)$ & $80(62.5)$ & $50(40.3)$ & & $11(22.4)$ & \\
\hline$>60$ years & $122(48.4)$ & $48(37.5)$ & $74(59.7)$ & & 38 (77.6) & \\
\hline Lymphocyte count & & & & $<0.001$ & & $<0.001$ \\
\hline$>1.0 \times 10^{9} / \mathrm{L}$ & $143(56.7)$ & 91 (71.1) & $52(41.9)$ & & $16(32.7)$ & \\
\hline$\leq 1.0 \times 10^{9} / \mathrm{L}$ & $109(43.3)$ & 37 (28.9) & $72(58)$ & & $33(67.3)$ & \\
\hline LDH & & & & $<0.001$ & & 0.001 \\
\hline$\leq 250 \mathrm{U} / \mathrm{L}$ & 49 (19.4) & $38(29.7)$ & $11(8.9)$ & & 4 (8.2) & \\
\hline $250-500 \mathrm{U} / \mathrm{L}$ & $106(42.1)$ & 57 (44.5) & $49(39.5)$ & & $15(30.6)$ & \\
\hline$>500 \mathrm{U} / \mathrm{L}$ & 97 (38.5) & $33(25.8)$ & $64(51.6)$ & & $30(61.2)$ & \\
\hline Respiratory rate & & & & $<0.001$ & & $<0.001$ \\
\hline$\leq 20 \mathrm{~b} / \mathrm{m}$ & $126(50)$ & 95 (74.2) & $31(25)$ & & $3(6.1)$ & \\
\hline $21-25 \mathrm{~b} / \mathrm{m}$ & 39 (15.5) & 21 (16.4) & $18(14.5)$ & & $3(6.1)$ & \\
\hline $26-30 \mathrm{~b} / \mathrm{m}$ & $21(8.3)$ & $5(3.9)$ & 16 (12.9) & & $5(10.2)$ & \\
\hline$>30 \mathrm{~b} / \mathrm{m}$ & $66(26.2)$ & $7(5.5)$ & 59 (47.6) & & 38 (77.6) & \\
\hline Resting $\mathrm{O}_{2}$ saturation & & & & $<0.001$ & & $<0.001$ \\
\hline$\geq 95 \%$ & $77(30.6)$ & 63 (49.2) & $14(11.3)$ & & 1 (2) & \\
\hline $90-94 \%$ & $80(31.7)$ & $48(37.5)$ & $32(25.8)$ & & $7(14.3)$ & \\
\hline$<90 \%$ & $95(37.7)$ & 17 (13.3) & $78(62.9)$ & & $41(83.7)$ & \\
\hline A-a gradient & & & & $<0.001$ & & $<0.001$ \\
\hline Normal & $80(31.7)$ & $67(52.3)$ & $13(10.5)$ & & 0 & \\
\hline Elevated & $172(68.3)$ & $61(47.7)$ & 111 (89.5) & & $49(100)$ & \\
\hline 10 feet $\mathrm{O}_{2}$ desaturation test & & & & $<0.001$ & & $<0.001$ \\
\hline$<3 \%$ & $79(31.3)$ & $62(48.4)$ & $17(13.7)$ & & 2 (4) & \\
\hline$\geq 3 \%$ & $173(68.7)$ & $66(51.6)$ & 107 (86.3) & & $47(96)$ & \\
\hline Days in hospital & $10(7-12)$ & $8(6-11)$ & $11(9-13)$ & $<0.001$ & $10(8-12)$ & 0.60 \\
\hline
\end{tabular}

TABLE 2: Characteristics of enrolled patients and their correlation with progression of disease and mortality.

Skewedly distributed continuous variables are expressed as medians (interquartile range, IQR) and compared with Mann-Whitney U test; categorical variables are presented as $\mathrm{n}(\%)$ and compared by the chi-square test.

COPD, chronic obstructive pulmonary disease; CLD, chronic liver disease; CRF, chronic renal failure; SOB, shortness of breath; LDH, lactate dehydrogenase; b/m, breaths per minute; $\mathrm{HRCT}$, high resolution computed tomography; $\mathrm{O}_{2}$, oxygen; A-a gradient, alveolar to arterial oxygen gradient.

\section{Regression analysis and construction of nomogram}

Univariate and multivariate Cox regression analysis showed that 10 feet $\mathrm{O} 2$ desaturation test (HR: 0.99, 95\% CI: $0.95-1.04, p=0.706$ ) is not a powerful predictor of progression of disease; however, it substantiates that increasing respiratory rate (HR: $1.06,95 \%$ CI: $1.03-1.09, \mathrm{p}<0.001$ ), decreasing resting oxygen saturation (HR: 0.95, 95\% CI: 0.91-0.99, $\mathrm{p}=0.019$ ) and greater than normal A-a gradient (HR: 1.00, 95\% CI: $1.0-1.15, \mathrm{p}=0.022$ ) 


\section{Cureus}

are independent high-risk factors associated with progression of illness. Based on these factors, a predictive nomogram (Figure 1) was formulated, and each variable was categorized to express its weight of impact on progression of the illness.

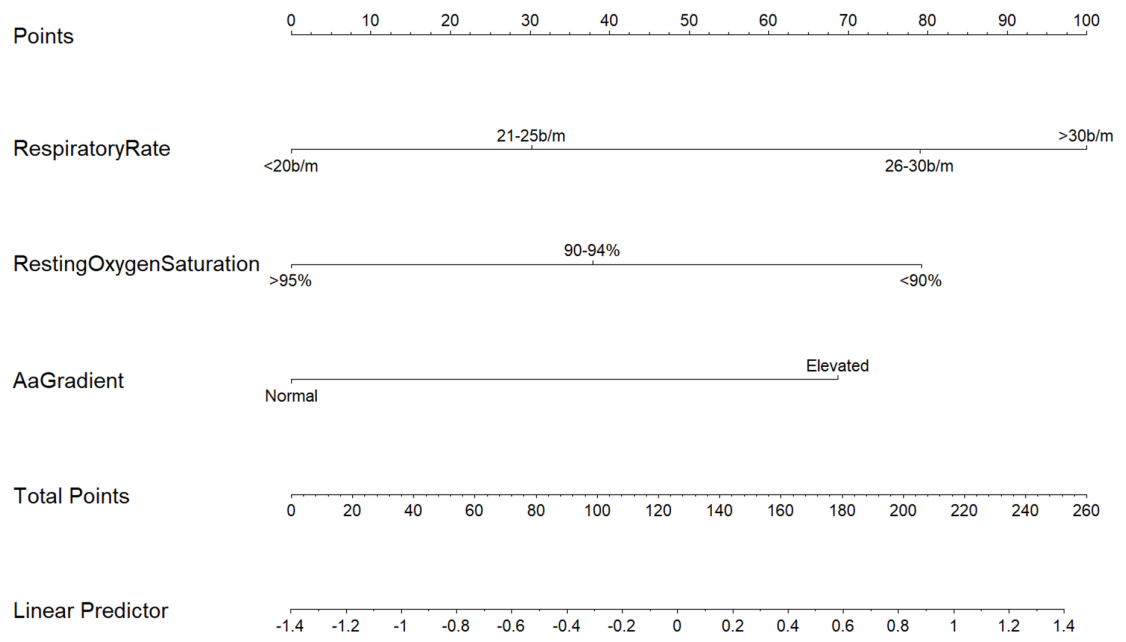

\section{FIGURE 1: Formulated nomogram for prediction of worsening disease.}

The value of each Respiratory Assessment Scoring (RAS) model variable is given a certain score on a point scale from 0 to 100 ; to utilize the nomogram, value of each variable is determined on corresponding axis, a line is drawn to the point axis, points from all the variables are added, and total points are projected to the linear predictor.

$\mathrm{b} / \mathrm{m}$, breaths per minute.

Respiratory rate was divided into four levels; less than and equal to $20 \mathrm{~b} / \mathrm{m}, 21$ to $25 \mathrm{~b} / \mathrm{m}$ (HR: 1.40, 95\% CI: 0.76-2.57), 26 to $30 \mathrm{~b} / \mathrm{m}$ (HR: 2.40, 95\%CI: $1.24-4.64$ ) and more than $30 \mathrm{~b} / \mathrm{m}$ (HR: 3.03, 95\%CI: 1.77-5.19). Resting O2 saturation was grouped as more than and equal to 95\%, 90\%-94\% (HR: 1.52, 95\% CI: 0.76-3.06) and less than $90 \%$ (HR: $2.41,95 \%$ CI: 1.15-5.06). For A-a gradient a value higher than normal for age was regarded as a cutoff (HR: 2.14, 95\% CI: 1.04-4.39), as illustrated in Table 3. 


\section{Cureus}

\begin{tabular}{|c|c|c|c|c|c|}
\hline Comorbidi & \multicolumn{2}{|l|}{$\begin{array}{l}\text { Univariate Cox analysis HR }(95 \% \mathrm{Cl}) \\
\text { Value }\end{array}$} & \multicolumn{2}{|l|}{$\begin{array}{l}\text { Multivariate Cox analysis HR }(95 \% \text { Cl) } \\
\text { Value }\end{array}$} & p- \\
\hline Without & 1 & - & 1 & _- & \\
\hline With & $0.97(0.66-1.43)$ & 0.876 & $0.89(0.59-1.33)$ & 0.564 & \\
\hline \multicolumn{6}{|c|}{ Age (Years) } \\
\hline$\leq 60$ & 1 & - & 1 & - & \\
\hline$>60$ & $1.87(1.30-2.68)$ & $<0.001$ & $1.50(1.02-2.22)$ & 0.041 & \\
\hline \multicolumn{6}{|c|}{ Lymphocyte (x $\left.10^{9} / \mathrm{L}\right)$} \\
\hline$>1.0$ & 1 & - & 1 & - & \\
\hline$\leq 1.0$ & $2.30(1.61-3.29)$ & $<0.001$ & $1.76(1.20-2.57)$ & 0.003 & \\
\hline \multicolumn{6}{|c|}{ LDH (U/L) } \\
\hline$\leq 250$ & 1 & - & 1 & - & \\
\hline $250-500$ & $2.43(1.26-4.68)$ & 0.008 & $2.17(1.13-4.20)$ & 0.020 & \\
\hline$>500$ & $4.09(2.15-7.75)$ & $<0.001$ & $3.33(1.74-6.37)$ & $<0.001$ & \\
\hline \multicolumn{6}{|c|}{ Respiratory rate $(\mathrm{b} / \mathrm{m})$} \\
\hline$\leq 20$ & 1 & - & 1 & - & \\
\hline $21-25$ & $2.21(1.24-3.95)$ & 0.007 & $1.40(0.76-2.57)$ & 0.281 & \\
\hline $26-30$ & $4.85(2.65-8.90)$ & $<0.001$ & $2.40(1.24-4.64)$ & 0.009 & \\
\hline$>30$ & $6.66(4.28-10.38)$ & $<0.001$ & $3.03(1.77-5.19)$ & $<0.001$ & \\
\hline \multicolumn{6}{|c|}{ Resting oxygen saturation (\%) } \\
\hline$\geq 95$ & 1 & - & 1 & - & \\
\hline $90-94$ & $2.57(1.37-4.82)$ & 0.003 & $1.52(0.76-3.06)$ & 0.239 & \\
\hline$<90$ & $7.83(4.41-13.89)$ & $<0.001$ & $2.41(1.15-5.06)$ & 0.020 & \\
\hline \multicolumn{6}{|c|}{ A-a Gradient } \\
\hline Normal & 1 & - & 1 & - & \\
\hline Elevated & $5.71(3.21-10.17)$ & $<0.001$ & $2.14(1.04-4.39)$ & 0.038 & \\
\hline \multicolumn{6}{|c|}{$\begin{array}{l}10 \text { feet } \mathrm{O}_{2} \text { desaturation test } \\
\text { (\%) }\end{array}$} \\
\hline$<3$ & 1 & - & 1 & - & \\
\hline$\geq 3$ & 3.89 (2.33-6.49) & $<0.001$ & $1.44(0.78-2.62)$ & 0.227 & \\
\hline
\end{tabular}

TABLE 3: Univariate and multivariate Cox proportional hazards regression analysis of progression of illness with CALL and RAS Score parameters.

HRs were calculated comparing without comorbidity versus with comorbidity, age $\leq 60$ years versus $>60$ years, lymphocyte count $>1.0 \times 10 / L$ versus $\leq 1.0 \times 10 / \mathrm{L}$, LDH $\leq 250 \mathrm{U} / \mathrm{L}$ versus LDH $250-500 \mathrm{U} / \mathrm{L}$ or $>500 \mathrm{U} / \mathrm{L}$, respiratory rate $\leq 20 \mathrm{~b} / \mathrm{m}$ versus $21-25 \mathrm{~b} / \mathrm{m}$ or $26-30 \mathrm{~b} / \mathrm{m}$ or $>30 \mathrm{~b} / \mathrm{m}$, resting oxygen saturation $\geq 95 \%$ versus $90 \%-94 \%$ or $<90 \%$, normal A-a gradient versus elevated A-a gradient, 10 feet $\mathrm{O}_{2}$ desaturation $<3 \%$ versus $\geq 3 \%$.

HR, hazard ratio; CALL, comorbidity, age, lymphocyte and LDH; RAS, respiratory assessment scoring; LDH, lactate dehydrogenase; b/m, breaths per minute; A-a gradient, alveolar arterial oxygen gradient; $\mathrm{O}_{2}$, oxygen. 


\section{Cureus}

CI: $1.02-2.22, \mathrm{p}=0.041$ ), lymphocyte count of less than $1.0 \times 109 / \mathrm{L}$ (HR: 1.76, 95\% CI: $1.20-2.57, \mathrm{p}=0.003$ ), LDH of 250-500 U/L (HR: 2.17, 95\% CI: 1.13-4.20, p=0.020), and LDH of more than $500 \mathrm{U} / \mathrm{L}$ (HR: 3.33, 95\% CI: 1.74-6.37, $\mathrm{p}<0.001$ ) were also high-risk elements linked with disease progression. On the other hand, presence of comorbidity revealed a $\mathrm{p}=0.565$ on multivariate regression for progression of illness.

\section{Model assessment}

Performance of the RAS model was checked using ROC curve where area under the ROC (AUROC) was $85 \%$ (95\%CI: 80\%-89\%) (Figure 2). A cutoff value of seven points was accepted for disease progression whose positive-predictive value $(95 \% \mathrm{CI})$ was $76 \%(68 \%-83 \%)$ and negative-predictive value $(95 \% \mathrm{CI})$ was $80 \%(73 \%$ 87\%) for prediction (Table 4). Using a cutoff value of nine points for mortality associated with the disease, the positive predictive value $(95 \% \mathrm{CI})$ was $55 \%(43 \%-67 \%)$ and the negative predictive value $(95 \% \mathrm{CI})$ was $95 \%$ (91\%-98\%) (Table 5).

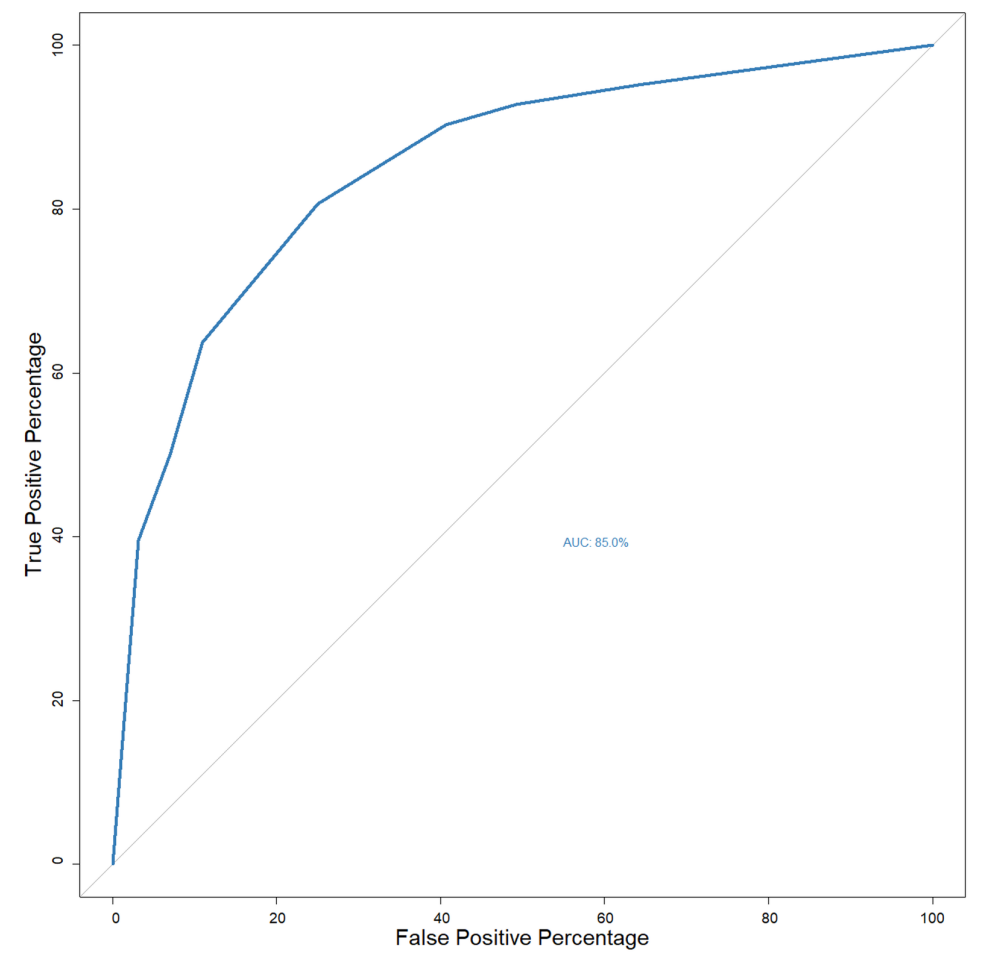

FIGURE 2: ROC curve correlating RAS Score with progression of illness.

ROC, receiver operating characteristics; RAS, respiratory assessment scoring; AUC, area under the curve. 


\section{Cureus}

\section{Variable}

AUROC, \% (95\% Cl)

Cutoff value

Sensitivity, \% (95\% Cl)

Specificity, \% $(95 \% \mathrm{Cl})$

Positive predictive value, $\%$ (95\% Cl)

Negative predictive value, $\%(95 \% \mathrm{Cl})$

Positive likelihood ratio $(95 \% \mathrm{Cl})$

Negative likelihood ratio $(95 \% \mathrm{Cl})$
Enrolled patients $(n=252)$

$85(80-89)$

$81(74-88)$

$75(68-83)$

$76(68-83)$

$80(73-87)$

$3.23(2.36-4.41)$

$0.26(0.18-0.38)$

TABLE 4: Accuracy of RAS Model for estimating risk of progression of disease.

RAS, respiratory assessment scoring; AUROC, area under the receiver operating characteristic curve; $\mathrm{Cl}$, confidence interval.

\section{Variable}

AUROC, \% (95\% Cl)

Cutoff value

Sensitivity, \% (95\% Cl)

Specificity, \% (95\% Cl)

Positive predictive value, $\%(95 \% \mathrm{Cl})$

Negative predictive value, $\%(95 \% \mathrm{Cl})$

Positive likelihood ratio $(95 \% \mathrm{Cl})$

Negative likelihood ratio $(95 \% \mathrm{Cl})$

\section{Enrolled patients $(n=252)$}

$90(86-94)$

9

$80(68-91)$

$84(79-89)$

$55(43-67)$

$95(91-98)$

$5.05(3.57-7.15)$

$0.24(0.14-0.42)$

\section{TABLE 5: Accuracy of RAS Model for estimating mortality.}

RAS, respiratory assessment scoring; AUROC, area under the receiver operating characteristic curve; $\mathrm{Cl}$, confidence interval.

Additionally, the RAS score was divided into three groups based on their chances to progression; scores of 36 points (class A) had less than 15\% probability of progression, 6-8 points were considered intermediate risk (class B) because of 15\%-50\% possibility of progression and high risk (class C) was appointed to set of 9-10 points with over $70 \%$ probability of worsening condition.

On day zero, $16.3 \%$ patients of the study fell in the CALL score category A, 38.1\% in B and $45.6 \%$ in C, among which $36.6 \%, 31.2 \%$, and $68.7 \%$ lead to progression of disease in A, B, and C categories, respectively. Likewise, on day zero 88 (34.9\%), 93 (36.9\%), and 71 (28.2\%) patients belonged to category A, B, and C on RAS score, respectively. Progression was observed in an increased number of patients in category C (87.3\%) followed by category B (53.8\%) and category A patients (13.6\%). Similarly, mortality figures were nonexistent in the RAS A group and considerably lower in RAS B (10.8\%) than the RAS C category (54.9\%).

\section{Discussion}

COVID-19 is a novel infectious disease that has spread throughout the world, resulting in over one million deaths in the course of a few months. Timely identification of patients who are at risk of progression to severe disease and mortality, can lead to better management and favorable outcomes. The purpose of this study was to appraise the potency of the CALL prognostic model [6] and to build an alternative scoring system based on the measurable respiratory parameters.

\section{Key findings}


The 252 COVID-19 patients in this study consisted of an increased amount of people of old age and with presence of comorbidities. Both of these groups of people were noted to have significant association with mortality. People who originally presented with shortness of breath and increased pulmonary infiltrates on radiographs were more prone to severe disease and mortality. Decreased lymphocyte count, raised LDH, increased RR, lower oxygen saturation at rest, elevated A-a gradient, oxygen desaturation on minimal exertion, and severe initial disease category also revealed a significant correlation with disease progression and death. In this study population, the CALL score proved to be a reliable prognosticator of worsening illness and associated fatality. However, multivariate regression analysis on variables of CALL score did not approve the presence of comorbidity to be a significant high-risk predictor of progression of the illness.

The Respiratory Assessment Scoring (RAS) system was developed based on three measurable high-risk respiratory parameters, namely; RR, resting oxygen saturation, and A-a gradient, which could identify a group of patients with either increased likelihood of stability, or worsening of disease and death. All three variables of the RAS model showed significant relation with the progression of disease on Cox regression studies.

\section{Comparison with existing literature}

The median age (IQR) for patients who died in this study was 70 (58-75) years, which is similar to many studies where older age is linked with rising mortality as evident in the narrative review by Stawicki et al. [7]. In conjunction with the results, lymphocyte count has been a remarkably well-validated variable employed in various studies, the lower count being an indicator of worse outcome [2,12-14]. Elevated LDH values have likewise been associated with a rise in odds of mortality, identical to findings in this study [15-17]. The presence of lung infiltrates was noticed to have a significant correlation with progression and death, which justifies its value in already established models [18-19].

In one study, the CALL score has turned out to have a good prognostic index for in-hospital mortality but not for disease progression [20]. However, a significant amount of patients with higher CALL scores in this study population developed progression of disease, as Ji et al. said that CALL score with a cut off value of nine points showed positive and negative predictive values of $78.3 \%$ and $11.9 \%$ respectively [6]. It was observed that the three variables of CALL score; age more than 60 years, lymphocyte count of less than $1.0 \mathrm{x}$ 109/L and increased LDH, were reliable predictors of worsening disease condition as put forth by Ji et al. [6]. In contrast, the presence of comorbidity alone was not found to be a reliable independent risk factor of disease progression in this sample of 252 patients, which falls in disagreement with many studies that previous comorbid conditions in coronavirus patients are correlated with worsening disease and mortality [2-4, 7, 21]. A few predictive models have also failed to establish comorbidity as a prognosticator of disease severity, suggesting a demand to properly establish and validate the effect of previous disease conditions in COVID-19 patients [22-23].

While several predictive models have been produced for COVID-19, only one scoring model has been found to integrate simple and clinically useful parameters such as increased respiratory rate and reduced oxygen saturation to assess disease progression, however, this said Brescia-COVID Respiratory Severity Scale (BCRSS) was a rapidly established score that has not yet been validated [19]. RAS model specifications, i.e. respiratory rate and oxygen saturation has been used as a response to noninvasive ventilation in COVID-19 patients $[19,24]$. These along with and A-a gradient have also been already validated to monitor respiratory failure in intensive care setups [25].

\section{Strengths and limitations}

The RAS model was used as an investigational score in this study. It has a high negative predictive value for mortality and fair negative and positive predictive values for disease progression. It can facilitate betterinformed discussions between clinicians and patients' families about the anticipated clinical trajectory, allowing accurate and timely advance-care planning.

This study was a pilot study for the RAS carried out at a single center with a number of limitations. Firstly, the sample size was small and secondly, a prospective study is also warranted to test the validity and efficacy of this model. Also, the 10 feet desaturation test that was devised and used, is not an otherwise validated tool for respiratory assessment. However, it has been found that in a substantial group of patients with COVID-19, hypoxia becomes apparent only on exertion [26]. Since some subjects in this sample population were already severely hypoxemic on rest, the validity of this test is also questionable. Nevertheless, due to the significant association of exertional desaturation observed with disease progression and fatality, it is paramount to provide a safe and effective minimal exertional desaturation test for coronavirus patients, also emphasized by Greenhalgh et al. [9].

\section{Conclusions}

The CALL and RAS scores achieved significant predictive power of disease progression and mortality in this study. Nonetheless, more prospective studies are required for validation of these scores, in order to justify 
their use in the setting of this devastating disease. Moreover, the importance and role of comorbidities and minimal exercise oxygen desaturation tests need to be further investigated to explain and validate their role in COVID-19 patients. The spectrum and implications of the novel coronavirus disease ought to be explored in detail and research on larger sample populations using multi-center studies is required to evince authentic knowledge.

\section{Additional Information \\ Disclosures}

Human subjects: Consent was obtained by all participants in this study. Ethical Review Committee (ERC) PEMH issued approval A/28/186/2020. The original article titled "CALL Score and RAS Score as Predictive Models for COVID-19" submitted by Dr. Sultan Mehmood Kamran and co-authors was reviewed in meeting of ERC held on 30th June 2020 at Pak Emirates Military Hospital. Committee has approved the project with consensus. Should any of aspects of work/project be changed, the matter should be brought to the board again for review. Please note that this grant of approval is for ethical aspects of work. Interim report on progress of the study should be submitted from time to time where applicable. . Animal subjects: All authors have confirmed that this study did not involve animal subjects or tissue. Conflicts of interest: In compliance with the ICMJE uniform disclosure form, all authors declare the following: Payment/services info: All authors have declared that no financial support was received from any organization for the submitted work. Financial relationships: All authors have declared that they have no financial relationships at present or within the previous three years with any organizations that might have an interest in the submitted work. Other relationships: All authors have declared that there are no other relationships or activities that could appear to have influenced the submitted work.

\section{Acknowledgements}

I would like to express my deep gratitude to Professor Dr. Muhammad Zafar Ali, Professor Dr. Amir Fakhar, Professor Dr. Khalid Raja, Dr. Kaswar Sajjad, Dr. Asad Zaman khan and Professor Dr. Malik Nadeem Azam for their patient guidance, enthusiastic encouragement and useful critiques of this research work. My grateful thanks are also extended to my colleagues; Dr. Yousaf Jamal, Dr. Kumail Abbass Khan, Dr. Bilal Ahmad, Dr. Fahd bin Haider, Dr. Maryam Hussain, Dr. Rizwan Azam, Dr. Farrukh Sher, Dr. Fatima, Dr. Saeed, Dr. Zulqernain, Dr. Moed, Dr. Naqeeb Ullah, Dr. Javeria, Dr. Zahra, Dr. Mehmood Hussain, and Dr. Komal for their enthusiastic encouragement and useful critiques of this research work.

\section{References}

1. WHO Coronavirus Disease (COVID-19) Dashboard / WHO Coronavirus Disease (COVID-19) Dashboard . (2020). Accessed: October 28, 2020: https://covid19.who.int/.

2. Guan W, Ni Z, Hu Y, et al.: Clinical characteristics of coronavirus disease 2019 in China . N Engl J Med. 2020, 382:1708-1720. 10.1056/NEJMoa2002032

3. Guan W-J, Liang W-H, Zhao Y, et al.: Comorbidity and its impact on 1590 patients with COVID-19 in China: a nationwide analysis. Eur Respir J. 2020, 55:2000547. 10.1183/13993003.00547-2020

4. Yang X, Yu Y, Xu J, et al.: Clinical course and outcomes of critically ill patients with SARS-CoV-2 pneumonia in Wuhan, China: a single-centered, retrospective, observational study. Lancet Respir Med. 2020, 8:475-481. 10.1016/S2213-2600(20)30079-5

5. Wynants L, Van Calster B, Collins GS, et al.: Prediction models for diagnosis and prognosis of covid-19: systematic review and critical appraisal. BMJ. 2020, 369:m1328. 10.1136/bmj.m1328

6. Ji D, Zhang D, Xu J, et al.: Prediction for progression risk in patients with COVID-19 pneumonia: the CALL score. Clin Infect Dis. 2020, 71:1393-1399. 10.1093/cid/ciaa414

7. Stawicki S, Jeanmonod R, Miller A, et al.: The 2019-2020 novel coronavirus (severe acute respiratory syndrome coronavirus 2) pandemic: A joint american college of academic international medicine-world academic council of emergency medicine multidisciplinary COVID-19 working group consensus paper. J Glob Infect Dis. 2020, 12:47-93.

8. Clinical management of COVID-19. (2020). Accessed: October 1, 2020: https://www.who.int/publications/i/item/clinical-management-of-covid-19.

9. What is the efficacy and safety of rapid exercise tests for exertional desaturation in covid-19? - CEBM . (2020). Accessed: October 5, 2020: https://www.cebm.net/covid-19/what-is-the-efficacy-and-safety-ofrapid-exercise-tests-for-exertional-desaturation-in-....

10. Ghasemi A, Zahediasl S: Normality tests for statistical analysis: a guide for non-statisticians . Int J Endocrinol Metab. 2012, 10:486-489. 10.5812/ijem.3505

11. Hantzidiamantis PJ, Amaro E: Physiology, Alveolar to Arterial Oxygen Gradient. StatPearls Publishing, Treasure Island, FL; 2019.

12. He Z, Zhao C, Dong Q, et al.: Effects of severe acute respiratory syndrome (SARS) coronavirus infection on peripheral blood lymphocytes and their subsets. Int J Infect Dis. 2005, 9:323-330. 10.1016/j.ijid.2004.07.014

13. Wang F, Nie J, Wang H, et al.: Characteristics of peripheral lymphocyte subset alteration in COVID-19 pneumonia. J Infect Dis. 2020, 221:1762-1769. 10.1093/infdis/jiaa150

14. Fathi N, Rezaei N: Lymphopenia in COVID- 19: therapeutic opportunities. Cell Biol Int. 2020, 44:1792-1797. 10.1002/cbin.11403

15. Shi J, Li Y, Zhou X, et al.: Lactate dehydrogenase and susceptibility to deterioration of mild COVID-19 patients: a multicenter nested case-control study. BMC Med. 2020, 18:168. 10.1186/s12916-020-01633-7

16. Henry BM, Aggarwal G, Wong J, et al.: Lactate dehydrogenase levels predict coronavirus disease 2019 
(COVID-19) severity and mortality: a pooled analysis. Am J Emerg Med. 2020, 38:1722-1726.

10.1016/j.ajem.2020.05.073

17. Liang W, Liang H, Ou L, et al.: Development and validation of a clinical risk score to predict the occurrence of critical illness in hospitalized patients with COVID-19. JAMA Intern Med. 2020, 180:1081-1089.

10.1001/jamainternmed.2020.2033

18. Guo L, Wei D, Zhang X, et al.: Clinical features predicting mortality risk in patients with viral pneumonia: the MuLBSTA score. Front Microbiol. 2019, 10:2752. 10.3389/fmicb.2019.02752

19. Duca A, Piva S, Focà E, Latronico N, Rizzi M: Calculated decisions: brescia-COVID respiratory severity scale (BCRSS)/algorithm. Emerg Med Pract. 2020, 22:CD1-CD2.

20. Grifoni E, Valoriani A, Cei F, et al.: The CALL score for predicting outcomes in patients with COVID-19 . Clin Infect Dis. 2020, 10.1093/cid/ciaa686

21. Wendel Garcia PD, Fumeaux T, Guerci P, et al.: Prognostic factors associated with mortality risk and disease progression in 639 critically ill patients with COVID-19 in Europe: initial report of the international RISC19-ICU prospective observational cohort. EClinicalMedicine. 2020, 25:100449. 10.1016/j.eclinm.2020.100449

22. Zhou F, Yu T, Du R, et al.: Clinical course and risk factors for mortality of adult inpatients with COVID-19 in Wuhan, China: a retrospective cohort study. Lancet. 2020, 395:1054-1062. 10.1016/S01406736(20)30566-3

23. Zhang C, Qin L, Li K, et al.: A novel scoring system for prediction of disease severity in COVID-19 . Front Cell Infect Microbiol. 2020, 10:318. 10.3389/fcimb.2020.00318

24. Sartini C, Tresoldi M, Scarpellini P, et al.: Respiratory parameters in patients with COVID-19 after using noninvasive ventilation in the prone position outside the intensive care unit. JAMA. 2020, 323:2338-2340. 10.1001/jama.2020.7861

25. Radermacher P, Maggiore SM, Mercat A: Gas exchange in acute respiratory distress syndrome. Am J Respir Crit Care Med. 2017, 196:964-984. 10.1164/rccm.201610-2156SO

26. Dhont S, Derom E, Van Braeckel E, Depuydt P, Lambrecht BN: The pathophysiology of "happy” hypoxemia in COVID-19. Respir Res. 2020, 21:198. 10.1186/s12931-020-01462-5 